\title{
Action Plan for Prolongation of Nephrogenesis in Preterm and Growth Restricted Babies: Explore Ultrastructure of the Nephrogenic Zone, Identify a Molecular Target, Select a Viable Drug and Find a Path for Administration
}

\author{
Author \\ Will W. Minuth
}

\author{
Affiliation \\ Institute of Anatomy, University of Regensburg, Regensburg, \\ Germany \\ Key words \\ preterm and low birth weight babies, fetal kidney, nephro- \\ genic zone, stem cell niche, impaired nephrogenesis, \\ morphogen, prolongation of nephrogenesis, drug delivery \\ received 04.05 .2017 \\ accepted 12.06.2017 \\ Bibliography \\ DOI https://doi.org/10.1055/s-0043-113637 \\ Published online: 12.7.2017 \\ Drug Res 2018; 68: 5-16 \\ (c) Georg Thieme Verlag KG Stuttgart · New York \\ ISSN 2194-9379 \\ Correspondence \\ Prof. Dr. Will W. Minuth \\ Institute of Anatomy \\ University of Regensburg \\ D-93077 Regensburg \\ Germany \\ Tel.: + 49/941/943 2820, Fax: + 49/941/943 2868 \\ will.minuth@vkl.uni-regensburg.de
}

\begin{abstract}
A large amount of investigations informs about primary steps of mammalian kidney development such as anlage of the organ and initial nephron formation, while only few data exists about the late phase of human kidney development. In particular, little attention was up to date addressed to the decrease of morphogenic activity in the nephrogenic zone short before birth and the vanishing of all stem cell niches aligned beyond the organ capsule. There is evidence that molecular controlling of this normal but degenerative developmental process also plays a decisive role in the kidneys of preterm and growth restricted babies. Although they are born in a phase of active nephrogenesis, a substantial percentage of them evolves oligonephropathy, formation of atypical glomeruli and immaturity of parenchyma. Pathologic findings point out that independent from chemical nature all suspected hampering influences sublimate in the nephrogenic zone. However, it is unknown, whether impaired nephrogenesis is locally caused by harming interstitial fluid, disturbance of morphogen signaling, unbalanced synthesis of extracellular matrix or limited cell to cell communication. Thus, first of all these issues must be resolved, then save application of medicines prolonging nephrogenesis waits for realization. Due to the unexpectedly complex microanatomy and physiology of the nephrogenic zone, it will be a particular challenge for the future.
\end{abstract}

\section{Introduction}

A lot of information is available dealing with development of the mammalian kidney. The major part of published literature was generated during the last years by an expanding repository of transgenic animals, progressing molecular and imaging techniques $[1,2]$. In most instances interest of research focused to the organ anlage and the primary formation of nephrons in mice kidney. In contrast, comparatively few data exists for the human developing kidney. This applies in particular for the late fetal period short before birth, when an unknown mechanisms switches off morphogenic activity in the nephrogenic zone. The change in developmental program is correlated with a loss of all stem cell niches aligned beyond the organ capsule so that formation of nephrons is terminated for the future live [3].
By the first view, the changes in developmental potential seem to be only of general biomedical interest. However, as well for preterm as low birth weight babies this subject is of special importance $[4,5]$. Although born in a phase of active nephrogenesis, a high percentage of them evolves oligonephropathy and prematurity of renal parenchyma causing severe restrictions for health later in life $[6,7]$. Various intra- and extrauterine influences seem to be responsible, but independent from chemical nature all noxious parameters converge finally to the nephrogenic zone and the here contained stem cell niches [8, 9].

An important research assignment is therefore to identify locally acting molecules impairing nephrogenesis, to find a strategy to antagonize their effects and to learn about drugs, which enable to prolong nephrogenesis by methods of modern regenerative med- 


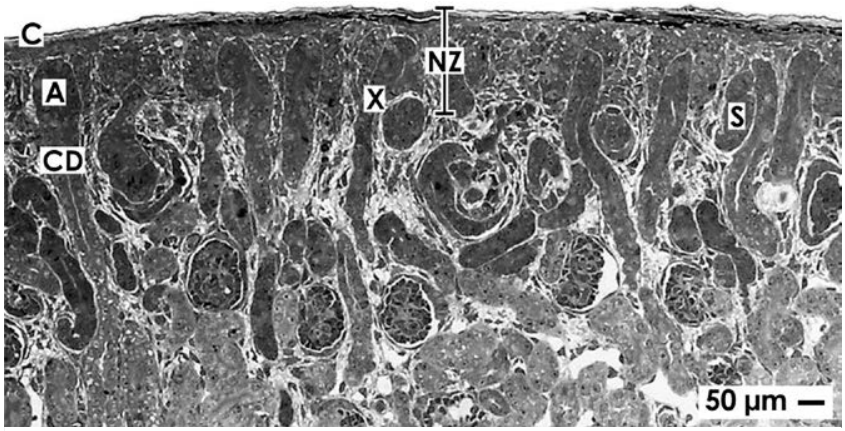

- Fig. 1 Semithin section of the nephrogenic zone (NZ) localized in the cortex cortices of a neonatal rabbit kidney. The histological section lines perpendicular to the organ capsule (C) and in parallel to lining collecting duct $(C D)$ tubules. Each of them exhibits a neck $(X)$ and forms at the ending a CD ampulla (A). At the lateral aspect of a $\mathrm{CD}$ ampulla renal vesicles and S-shaped bodies $(\mathrm{S})$ indicate active nephrogenesis.

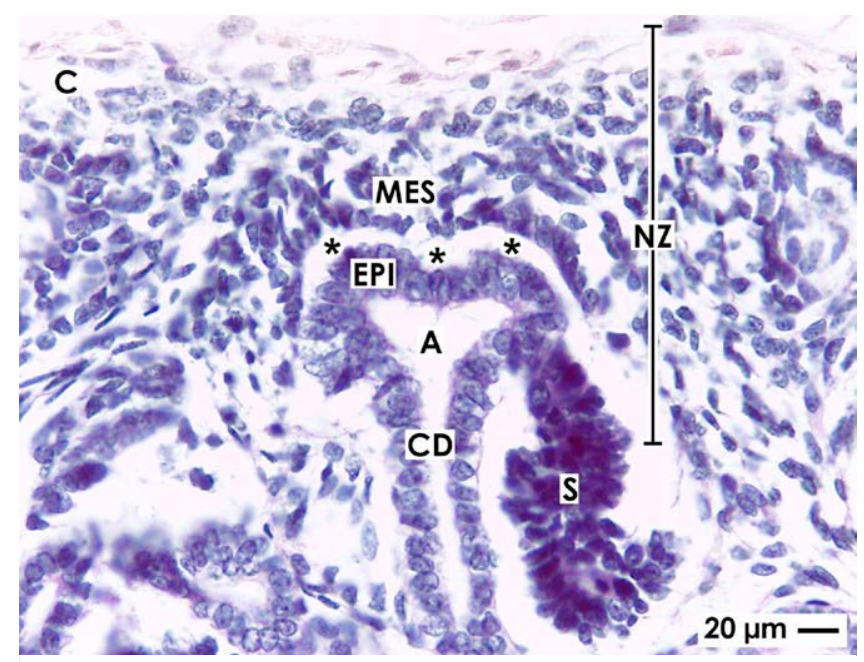

- Fig. 2 Details of the nephrogenic zone (NZ) and a stem cell niche in fetal human kidney shown by optical microscopy. A niche is covered by the capsule (C). Epithelial (EPI) stem cells are enclosed in the tip of an ampulla (A) at the end of a collecting duct (CD) tubule. Mesenchymal (MES) and epithelial stem cell bodies are separated by a striking interface (asterisks). S: developing S-shaped body.

icine $[10,11]$. However, development of a concept for recovery of the nephrogenic zone and contained stem cell niches is more difficult than commonly believed. First, so far no systematic analysis was performed to work out ultrastructural aspects of the nephrogenic zone in a human fetal kidney. Second, due to an incomplete vascular supply an effective access for an administered drug via the capillary system is unknown. Third, molecular aspects concerning morphogen transport and molecular signaling have to be adapted from animal species without knowing, whether beside homologous mechanisms also human-specific differences exist.

Without interpreting ethical issues, treatment of impaired nephrogenesis in a preterm infant principally could be implemented either by selected morphogens and related molecules using an innovative drug delivery system [12] or by an implantation of stem/ progenitor cells into the subcapsular space of the kidney [13]. Con- sequently, detailed knowledge is required about the distribution of an administered drug in the nephrogenic zone, binding on locally existing extracellular matrix, bioavailability on the site of niches, reaction on the target, metabolism and possible embryo-toxic side effects on neighboring maturing parenchyma. The same applies to correct implantation, survival, seeding and integration of stem cells in existing operational structures. Besides all this, one has to ask the question, whether the correct generative support of implanted stem cells is provided on demand. The actual problem is that the necessary information does not exist for a human fetal kidney. For this reasons, earlier findings from animal experments must help for the moment to explain some details. It is obvious that only an intense research program over years can answer raised human-specific issues. To facilitate the entry in that complex matter, elected features of the nephrogenic zone in context with drug delivery shall be presented here.

\section{Coordinates for Orientation}

The nephrogenic zone of a fetal mammalian kidney straddles as a band beyond the organ capsule and shows a width of up to $100 \mu \mathrm{m}$ depending on species [14]. In histological sections stained by hematoxylin-eosin solution it exhibits a slightly pronounced color profile [15]. For this reason, the nephrogenic zone is termed in single cases 'blue strip'.

From top to base, the nephrogenic zone is not a random accumulation of stem cells but it is specifically structured. The covering organ capsule consists of a tunica fibrosa and muscularis ( $\triangleright$ Fig. 1). Contained smooth muscle cells form here a unique tunnel system filled with fluid [16]. When exactly orientated sections are analyzed, beyond the inner side of the capsule only 2 layers of mesenchymal stem cells are visible. The inner layer of them is separated by an interface from tips of ureteric bud-derived CD ampullae containing epithelial stem cells ( $\triangleright$ Fig. 2). Further on, each tip of the aligned $\mathrm{CD}$ ampullae points towards the capsule. At the lateral aspect of a CD ampulla condensed mesenchyme, renal vesicles or S-shaped bodies are visible reflecting morphological signs of active nephrogenesis. Slightly further down, the dilated part of a CD ampulla continuous to a neck and then to a shaft that is connected with a with a differentiating $C D$ tubule [17]. This area defines the inner limit of the nephrogenic zone.

\section{Definition of Niche Sites}

Surprisingly, not only in the nephrogenic zone but also in the entire covering organ capsule stem cells are contained [18, 19]. When both areas are regarded as one claim, as well parenchyma as stroma are developing by this source of cells. Consequently, the nephrogenic zone and the capsule form per definition an extended niche area that covers the entire organ.

In contrast, formation of human renal parenchyma is driven by branching morphogenesis that produces during the late fetal period in elongating CD tubules bifid branches [20,21]. These occur in close vicinity to the organ capsule, where they dilate to form CD ampullae ( $\vee$ Fig. 2). As mentioned, their tips are always orientated towards two layers of nephrogenic mesenchymal stem cells and the covering capsule. During the process of nephron induction the 


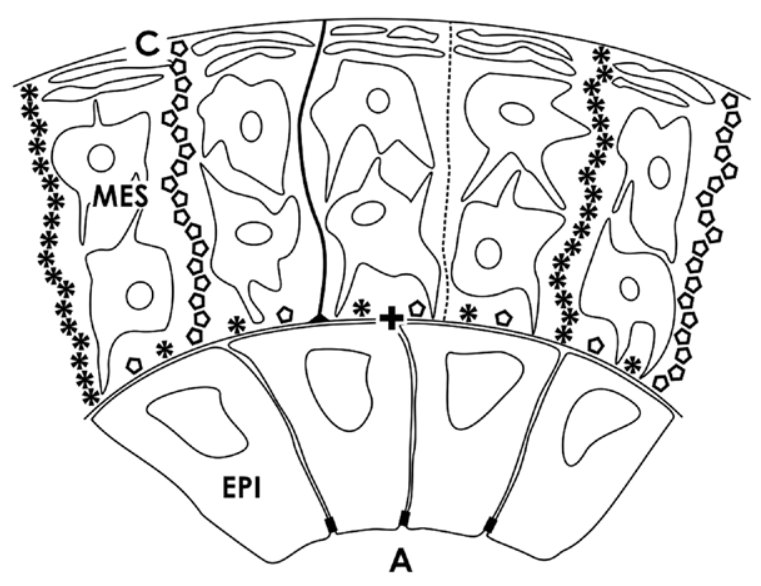

Fig. 3 Schema shows microfibers linking a stem cell niche with the inner side of the capsule. The basal lamina of a CD ampulla (A) tip is labeled by a cross (+). At this site microfibers binding Soybean Agglutinin (SBA; black line) and anti-collagen type I (black asterisks), type II (light circles), and type III (dotted line) originate, cross the interface, line through the 2 layers of mesenchymal stem cells to be linked on the organ capsule (C). (EPI) epithelial, (MES) mesenchymal stem cells.

tip of each CD ampulla including epithelial stem cells moves towards the inner layer of mesenchymal cells. Hence, only the tip of a single $C D$ ampulla with contained epithelial cells and some of the nearest $\mathrm{GDNF}^{+} / \mathrm{Six2}{ }^{+} / \mathrm{CITED} 1^{+}$mesenchymal cells are regarded as an individual renal niche $[8,22]$.

\section{Mounting of a Niche}

For the human fetal kidney no data are available, but for the neonatal rabbit kidney it was shown that each of these niches is mounted via microfibers with the organ capsule ( $\triangleright \mathbf{F i g}$. 3) [16]. Label by anti-collagen type I, II, III and Soybean agglutinin (SBA) depicts that microfibers originate on the basal lamina covering the tip of a $C D$ ampulla. Those line through both layers of mesenchymal stem cells to fasten on the inner side of the capsule. Presence of these microfibers illustrates that epithelial and mesenchymal stem cells do not meet by accident during induction of a nephron but are integral part of a special mounting. It further ensures that all of the niches stand in a certain lateral and vertical order along the inner side of the organ capsule.

Actual morphological data show that epithelial and mesenchymal stem cell bodies are not in direct contact but stand at a distance between 1 to $2 \mu \mathrm{m}$ ( $\triangleright$ Fig. 4) $[23,24]$. The resulting interface between was already earlier observed by optical microscopy [2528] and transmission electron microscopy [29-32]. It was illustrated in mice, rat, rabbit and human developing kidney, however, no further attention was directed to this spatial structure.

On neonatal rabbit kidney it was further shown that the basal aspect of epithelial stem cells is covered by a consistent basal lamina including a lamina fibroreticularis that exhibits a conspicuous fiber meshwork ( $\triangleright$ Fig. 5a) [33]. Special fixation of specimens in GA

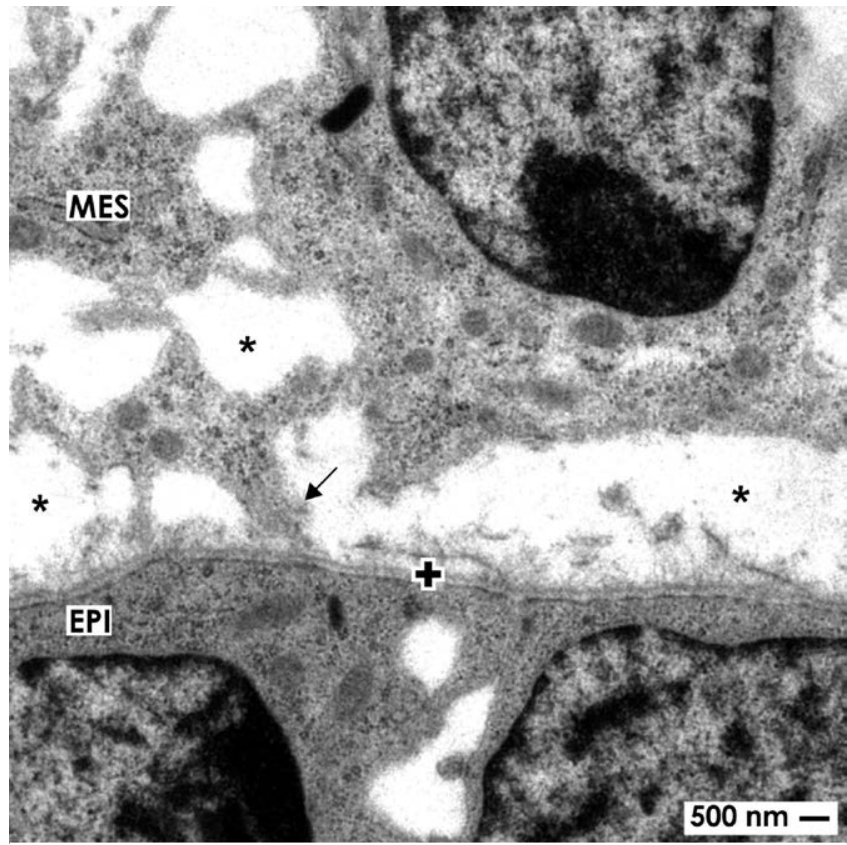

- Fig. 4 Transmission electron microscopy of a stem cell niche in neonatal rabbit kidney after fixation in glutaraldehyde solution. A clear interface (asterisk) separates mesenchymal (MES) and epithelial (EPI) stem cell bodies. However, projections (arrow) of mesenchymal cells cross the interface to contact epithelial cells. The basal lamina of epithelial cells at the tip of a CD ampulla is labeled by a cross $(+)$

solution including cupromeronic blue (CMB) reveals that numerous braces of proteoglycans are contained in the basal lamina and the interface separating mesenchymal and epithelial stem cell bodies ( $\triangleright$ Fig. 5b). Proteoglycans also occur on the surface of mesenchymal cell projections crossing the interface [34]. Moreover, specimens fixed in GA solution including ruthenium red (RR; $\triangleright$ Fig. $\mathbf{5 c}$ ) or tannic acid (TA; • Fig. 5d) unmasks previously not supposed textured extracellular matrix within the interface [35].

When impairment of nephrogenesis in the kidney of preterm or growth restricted babies is under analysis, both remodeling of illustrated microfibers and textured extracellular matrix at the interface of the niches has to be considered ( $\triangleright$ Fig. 4 and $\triangleright \mathbf{5}$ ). For example, tissue transglutaminases (TGases), matrix metalloproteinases (MMPs) and membrane targeted MMPs (MT-MMPs) are key mediators of this important process $[36,37]$. An equilibrated activity of TGase controls growth factor-stimulated signaling and in turn cell proliferation [38, 39]. It remains to work out, whether imbalance of TGase activity on illustrated microfibers or on extracellular matrix at the interface influences cell biological activity within renal stem cell niches of preterm infants. In the case an increased activity occurs, inhibitors of TGases may help to find back to an equilibrium between synthesis and degradation [40].

\section{Contacts Between Stem Cells}

Actual morphological data exhibits that masked extracellular matrix of the interface acts as a spacer to separate the bodies of mesenchymal and epithelial stem cells within a renal niche ( $>$ Fig. 4 and 5). 


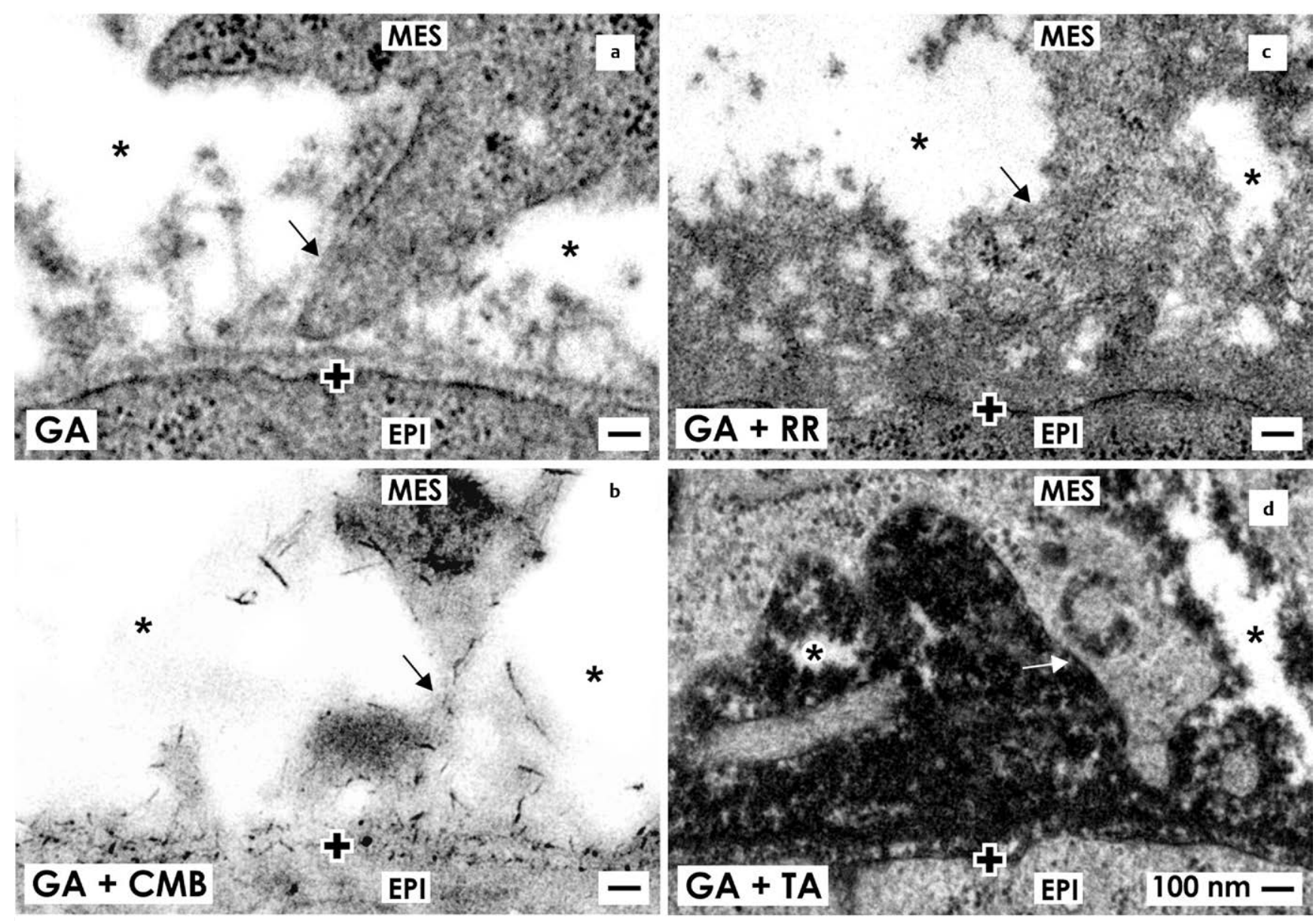

- Fig. 5 Transmission electron microscopy of the stem cell niche in neonatal rabbit kidney illustrates projections (arrow) of mesenchymal (MES) cells, which cross the interface (asterisk) to contact epithelial (EPI) cells. a Specimens fixed by conventional glutaraldehyde (GA) solution suggest that the interface is void. $\mathbf{b}$ Fixation by glutaraldehyde solution including cupromeronic blue (CMB) shows that numerous braces of proteoglycans are recognized on the surface of cell projections and within the basal lamina of epithelial stem cells. c Specimens fixed by GA solution including ruthenium red (RR) or $\mathbf{d}$ tannic acid (TA) unveil masked textured extracellular matrix at the interface. The basal lamina at the tip of a CD ampulla is labeled by a cross $(+)$.

Further projections (also called cytonemes or signaling filopodia) of mesenchymal cells cross the interface to contact the basal lamina of epithelial stem cells at the tip of a CD ampulla. Immunohistochemistry earlier showed that in a mesenchymal cell projection integrin $\alpha 8 \beta 1$ is present, which binds to nephronectin in the basal lamina of epithelial cells [41-43]. At this site also the micro-tubuledependent motor protein kinesin KIF26B was detected that is involved in regulating attraction, signal transduction and developmental patterning $[44,45]$.

Transmission electron microcopy further reveals that a projection penetrates the basal lamina at the tip of a CD ampulla $[24,46]$. At the end of a projection extracellular matrix forms a sleeve to ensure the contact. Further tunneling nanotubes occur between the end of a projection, the basal lamina and the basal plasma membrane of an epithelial cell ( $\triangleright$ Fig. 6). This important finding reveals that a path for cell to cell communication and transport of molecules via tunneling nanotubes exists [47]. It is obvious that disturbance of transport in tunneling nanotubes between mesenchymal and epithelial stem cells will have an impeding influence on this communication during induction of a nephron.

\section{Morphogens Control Niches}

Morphogens control the nephrogenic zone and contained stem cell niches. In an intact human kidney morphogens are active from organ anlage up to the birth [11]. Short before, morphogenic processes are downregulated and the last generation of niches aligned beyond the capsule is vanishing by an unknown molecular mechanism. However, so long as nephrogenesis is active, morphogens influence in a hierarchical manner a series of quite different developmental processes [48]. For example, morphogen Mdm2 fulfills in this orchestra a more general function such as supervision of survival, proliferation and competence of renal stem cells. Its positive signaling provokes expression of typical markers such as Amphiphysin, Cited1, Sall1 and Pax 2 [49].

More specialized tasks fulfill morphogens, which enable induction of a nephron $[50,51]$. It starts, when a branch of an elongating CD tubule forms a CD ampulla [52]. Only for a certain frame of time the nearest layer of $\mathrm{GDNF}^{+} \mathrm{Six}{ }^{+} \mathrm{CITED}^{+}$mesenchymal stem cells is exposed to the basal side of epithelial stem cells contained in the tip of a CD ampulla. Yet a reciprocal signaling of morphogens such Glial cell line-Derived Neurotrophic Factor (GDNF), Bone Mor- 


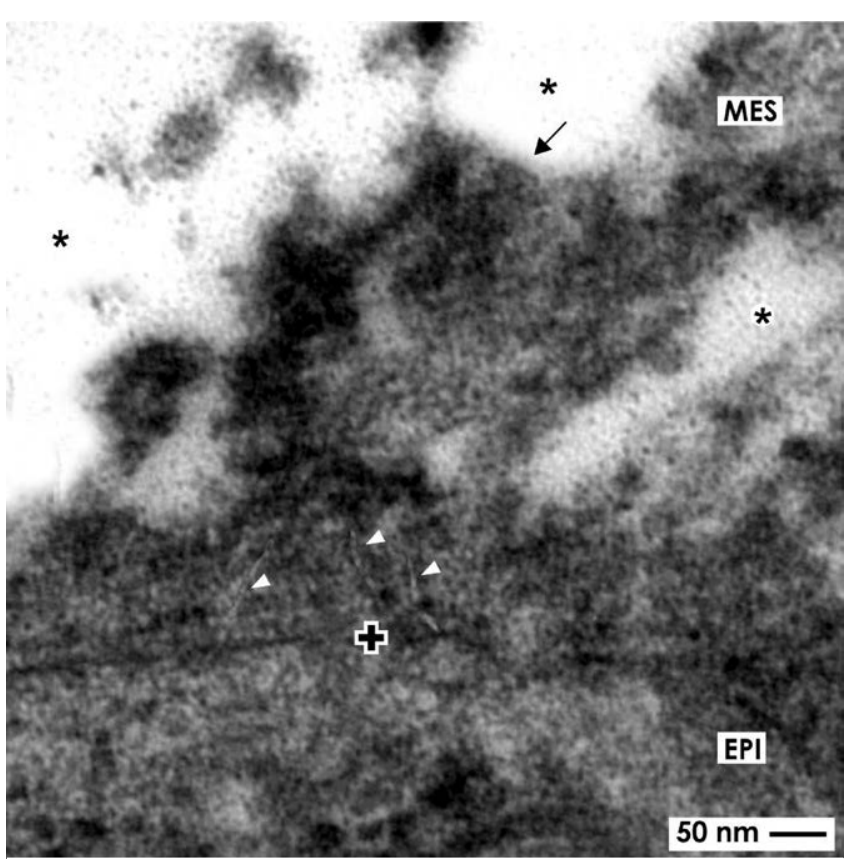

- Fig. 6 Transmission electron microscopy of the stem cell niche in neonatal rabbit kidney shows cell to cell contacts via tunneling nanotubes. A projection (arrow) of a mesenchymal (MES) cell is crossing the interface (asterisk) to perform a communication with epithelial (EPI) cells via tunneling nanotubes (arrow heads). The basal lamina of epithelial cells within the tip of a CD ampulla is marked by a cross $(+)$.

phogenetic Proteins (BMP4, BMP7), WNT family members (Wnt4, Wnt5a, Wnt9b) and Fibroblast Growth Factor (FGF8) takes place [53-58]. For an effective transmission of these signals receptors such as Fgfr1, Fgfr2, Gfra1, Notch 2, Ret tyrosine kinase receptor and transcription factors such as BRN1, FoxC2, LIMI, Osr1, Sall1, Pax2 and Wt1 must be activated [48].

When the signaling of these morphogens was successful, induced $\mathrm{GDNF}^{+} \mathrm{Six} 2{ }^{+} \mathrm{CITED}^{+}$cells separate, migrate and aggregate on the lateral side of a related $C D$ ampulla. Here they perform a mesenchyme-to-epithelial transition (MET) to develop into a renal vesicle and then a S-shaped body as first visible signs of a developing nephron [59].

\section{Unsettled Transport of Morphogens}

During the last years, transgenic animals and related experimental tools gave basic insights in morphogen signaling during nephrogenesis [60]. For example, by knocking down a gen for morphogen synthesis its missing action leads to restricted development. However, looking to the last link in the chain of molecular actions, there is nothing said about primary links such as regulation of morphogen synthesis, processing, secretion, binding on extracellular matrix and transport from one cell to the other. Due to missing information over time, the general impression appeared that all of the mentioned morphogens in the renal niche have more or less the same biophysical properties and that all of them reach their target by diffusion. As we know yet, this assumption is definitively wrong. Considering a therapeutic prolongation of nephrogenesis in pre-

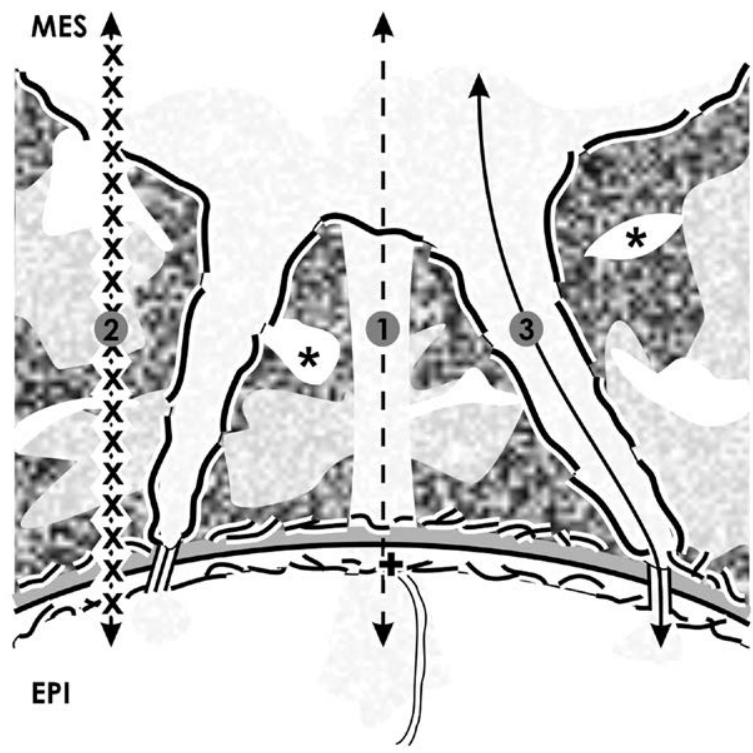

Fig. 7 Schematic illustration informs about 3 possible routes for the transport of morphogens within the renal stem cell niche. Mesenchymal (MES) and epithelial (EPI) cells are separated by an interface (asterisk) including textured extracellular matrix. Projections of mesenchymal cells cross it to establish a communication via tunneling nanotubes. It is supposed that morphogens with good solubility are transported by diffusion (1). Morphogens with minor solubility are secreted in interstitial fluid, bound on extracellular matrix, stored and provided on demand (2). Morphogens with poor solubility are transported in cell projections and tunneling nanotubes (3). The basal lamina of epithelial stem cells at the tip of a CD ampulla is marked by a cross $(+)$.

term or low birth weight babies, it is now time to reinvestigate regulation and transport of morphogens in the nephrogenic zone and contained stem cell niches for finding a suitable strategy for drug delivery.

To obtain concrete information about transmission of the morphogen signal, site-specific features of the renal stem cell niche were simulated in transfilter culture experiments. For example, morphogen Wnt4 expressing NIH3T3 cells were mounted for tubule induction on one side, while isolated nephrogenic mesenchyme was placed on the other side of a filter. Experiments demonstrated that separating filters with pore sizes of $0.1 \mu \mathrm{m}$ and above support induction of tubule formation, while pores of $0.05 \mu \mathrm{m}$ abolish it. By the way, morphogens are so small that a pore of this size is not an obstacle during transport. Surprisingly, solubilized molecules in form of a supernatant from Wnt4 expressing cells were not able to induce formation of tubules [61]. This unexpected result points out that the transport of a morphogen during induction of a nephron is not as easy as it looks, cannot be explained alone by diffusion and seems to act via contacting cell projections [62]. Also earlier morphological findings in niches of embryonic kidney [63] and further transfilter culture experiments [64] contradict the general assumption that all morphogens reach their target by diffusion.

To shed some light on the complex transport matter, one has first to bear in mind that each sort of involved morphogens has in- 
dividual biophysical properties. Second, action of different sorts of morphogens must be merged with the peculiar microarchitecture of the nephrogenic zone and contained niches ( $>$ Fig. 7). Third, label by cupromeronic blue depicts that within the interface, on the surface of cell projections and on the basal lamina proteoglycans are contained that can principally influence transport by selective binding of morphogens [65]. Finally, communication between mesenchymal and epithelial cells via projections respectively tunneling nanotubes points out that a route for a dosed transport of various molecules including morphogens principally exists [66].

\section{Function Encounters Structure}

Detailed knowledge about molecular and biophysical properties of involved morphogens is required, when a therapeutic prolongation of nephrogenesis in the kidneys of a preterm infant is under consideration. In the past it was fairly easy to suppose that the distance between mesenchymal and epithelial stem cells during nephron induction is minimal and that involved morphogens have the same good solubility in saline solution [48, 52]. Regarding these terms, the route for a diffusing morphogen is short and loss by dilution in interstitial fluid will be unattended small. In turn, a sharp gradient is building up, so that an effective concentration of a morphogen will reach the related receptor [67]. All of that sounds convincing but was not verified for the renal stem cell niche.

To obtain better insights, merging between the complex microarchitecture of the nephrogenic zone and the different biophysical features of involved morphogens helps. When morphogens are sorted in good [7, 22, 53], minor [68-70] and poor [71-73] solubility in saline solution, their transport can be allocated to recently detected morphological details ( $\triangleright$ Fig. 7). Since concrete data for the human renal stem cell niche is not available, the here introduced concept is founded on neonatal rabbit kidney [74, 75] and cell biological data raised in other developmental systems [76].

\section{Morphogens Reach Target by Diffusion}

Special fixation of specimens with glutaraldehyde (GA) solution including cupromeronic blue, ruthenium red respectively tannic acid for transmission electron microscopy demonstrates that extended areas of the interface within the renal stem cell niche contain filigree extracellular matrix $[74,75]$. In contrast, a complementary but much minor space does not show any label, appears to be filled only by interstitial fluid and is consequently best suited for diffusion of a readily soluble morphogen (Fig. 7.1). Due to its biophysical features, GDNF is an ideal candidate for transport this way. The molecule contains 134 amino acids, is secreted as a glycoprotein and is therefore readily soluble in interstitial fluid [77]. It may surprise, but only GDNF synthesized by mesenchymal stem cells was up to date defined in literature as a long-distance diffusible morphogen that binds on Ret tyrosine kinase receptor and a co-receptor GFR 1 localized at the tip of a CD ampulla $[22,78]$.

\section{Morphogens Meet Extracellular Matrix}

Cupromeronic blue label on mesenchymal cell projections and on the basal lamina of epithelial stem cells illustrates syndecans and/ or glypicans ( $\triangleright$ Fig. $\mathbf{5 b}$ ), while label by ruthenium red ( $\triangleright$ Fig. $\mathbf{5 c}$ ) or tannic acid ( $\vee$ Fig. $\mathbf{5 d}$ ) points to perlecans and other proteins of extracellular matrix within the interface of the niche [79]. Earlier published literature shows that proteoglycans are special modulators of kidney development interacting with GDNF, molecules of the FGF and TGF $\beta$ superfamilies, EGF receptor ligands and HGF [80-83]. The general concept is that binding of related morphogens on proteoglycans acts as a 'morphogenic switch' that influences as an inhibitor or facilitator the fine-tuning of a morphogen gradient between interacting cells (Fig. 7.2). The special meaning of extracellular matrix is recognized by the fact that environment lacking heparan sulfate proteoglycans (HSPGs) does not support formation of an effective Wnt gradient abolishing in turn further development [79].

\section{Morphogens Stay on Cells}

Morphogens Wnt4, Wnt5a and Wnt9b have an influence on renewal and differentiation of nephron progenitors, CD ampulla branching and nephron induction $[48,84]$. However, quite rarely information is given that Wnt molecules have posttranslational modifications in form of a saturated palmitic acid and an unsaturated palmitoleic acid so that they resemble lipoproteins with a poor solubility in interstitial fluid [72]. For that reason it appears most probable that they are not sprayed within the interstitial interface for diffusion but are secreted by epithelial cells only in the vicinity of mesenchymal cell projections (Fig. 7.3). In such a case they reach the plasma membrane of the target cell, where they can bind to a cargo that is able to transport Wnt molecules $[85,86]$. For example, in Drosophila Tkv-GFP receptor puncta are present in cell projections that move here in an anterograde or retrograde direction [87].

Morphogen Sonic Hedgehog (Shh) controls renal patterning [88]. This sort of molecule is not sprayed into the interstitial space but is produced in form of a particle that remains associated during transport with the cell surface on cell projections [89, 90]. Also the group of Bone Morphogenic Proteins (BMPs) belongs to morphogens with poor solubility in interstitial fluid [22]. Due to biophysical characteristics the transport of a BMP molecule through the interface by diffusion is unlikely. Instead, transport of BMPS at the contact site between a mesenchymal cell projection and an epithelial cell seems to be more probable. At those sites a BMP molecule can bind at the plasma membrane [91] and will be transported then with its receptor via microtubules [92].

\section{Transport of Morphogens Via Tunneling Nanotubes}

Actual literature demonstrates that projections of mesenchymal stem cells cross the interface and the adjacent basal lamina to establish a contact with epithelia stem cells at the tip of a CD ampulla ( Fig. 4) [74, 75]. Astonishingly, between the end of a projection and the basal plasma membrane of an epithelial cell tunneling nanotubes occur to establish a cell to cell communication ( $\vee$ Fig. 6). This path is also suitable for transport of organelles, membrane compounds and other kinds of molecules [93-98]. Although yet not proven, it appears most likely that also morphogens maintaining stemness and triggering nephron induction are transported 
this way at the right time, correct site and in a dosed amount (Fig. 7.3). However, up to date those transport functions in combination with tunneling nanotubes are not known for the renal stem cell niche. Instead they were investigated by in vitro experiments with renal cells in different context $[47,99]$. In sum, due to mentioned arguments and many unanswered questions the transport of morphogens within the renal niche via tunneling nanotubes waits to be investigated, when prolongation of nephrogenesis in preterm or low birth weight babies is therapeutically intended $[5,75,100]$.

\section{Shuttling of Morphogens Via Vesicles}

Beside mentioned routes, the intercellular transport of morphogens may also principally take place by vesicles such as exosomes (40$100 \mathrm{~nm}$ ) or microvesicles (100-1000 nm) [101, 102]. In those vehicles as well mRNA, microRNA as morphogens can be shuttled. $[103,104]$. However, up to date literature does not inform about transport of morphogens by vesicles within the renal stem cell niche.

\section{Incomplete Vascular Supply}

Due to running nephrogenesis one would assume that the nephogenic zone is provided from the medullary side by a perfect capillary system. However, the contrary is true. Vascular supply on this site is incomplete, and it was up to date not thoroughly examined. Histochemistry using Ulex europaeus I lectin on human fetal kidneys demonstrated that in the area of starting nephrons not intact capillaries but only endothelial cell strands exist [105]. In the neonatal rabbit kidney immunohistochemistry exhibited that capillaries line from cortical radiate arteries towards the nephrogenic zone. Vessels under construction occur on developing glomeruli. Further strands of endothelial cells line to the lower cleft of S-shaped bodies, where the glomerular tuft is arising ( $\mathbf{F i g . ~ 8 )}$. Only some endothelial cells occur at the lateral aspect of CD ampullae [106]. However, at the niche site including the tip of a CD ampulla and neighboring mesenchymal stem/progenitor cells, endothelial cells are not present.

For the rat kidney it was shown that expression of endothelial nitric oxide synthase takes place on developing S-shaped bodies but not within the mesenchymal cell layers [107]. An actual investigation on developing mice kidney exhibits that forming vessels at the nephrogenic zone are not flowed, although oxygenation is able to drive nephron progenitor differentiation [108]. Interestingly, an alternative spring for nutrition and oxygen might be that capillaries within the capsule produce interstitial fluid that is transported in the before mentioned but not carefully investigated tunnel system within the tunica muscularis of the organ capsule [16].

Hence, when a drug or a morphogen for prolongation of nephrogenesis is administered by infusion via the renal arterial vessel system, it will reach only the inner limit of the nephrogenic zone corresponding with developing glomeruli, late $\mathrm{S}$-shaped bodies and sites of CD tubule differentiation $[17,106]$. On the lateral aspect of $\mathrm{CD}$ ampullae capillaries are under construction, but on niches represented by the tip of a $C D$ ampulla and neighboring $\mathrm{GDNF}^{+} \mathrm{Six} 2^{+} \mathrm{CITED}^{+}$mesenchymal stem cells a perfused capillary system is not established. In turn, medication can reach this area only by long distance diffusion via interstitial fluid.

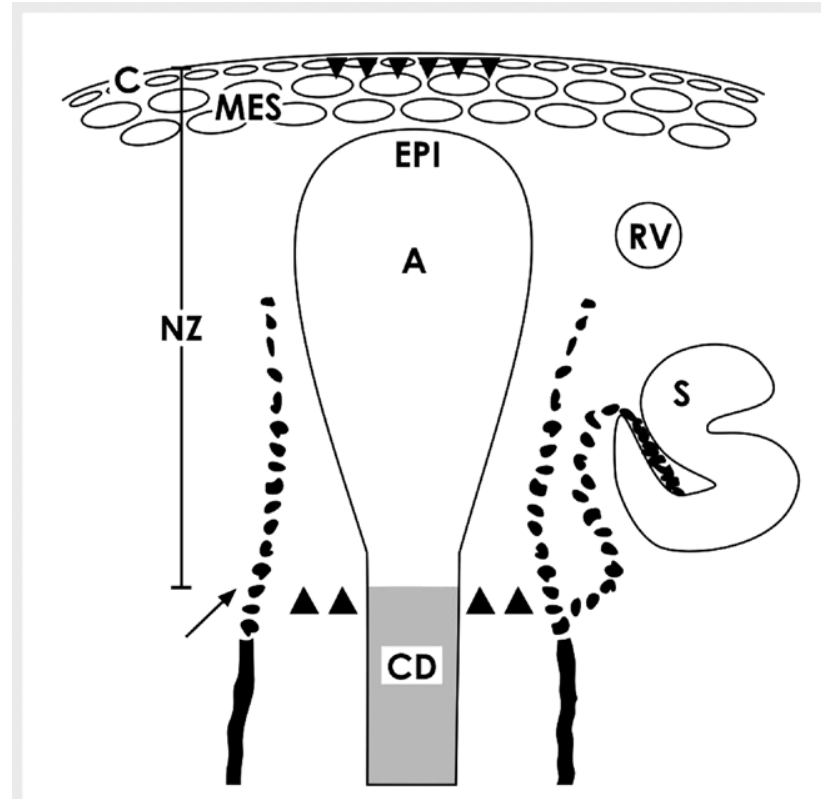

Fig. 8 Schematic illustration informs about the incomplete vascular supply of the nephrogenic zone (NZ) analyzed in neonatal rabbit kidney. Label by antibody EC1 [106] depicts that capillaries arise from cortical radiate arteries (arrow) lining in parallel to collecting duct (CD) tubules. Endothelial cells migrate to the lateral aspect of a CD ampulla (A) and to the lower cleft of a S-shaped body (S). In addition, cells of the tunica muscularis within the organ capsule (C) form intra- and extracellular tunnels (small arrow heads) [16]. It is obvious that the center of a niche comprising the tip of a CD ampulla with epithelial (EPI) cells, the interface and neighboring mesenchymal (MES) cells is avascular. Only via the rete capsularis and tunnels (small arrow heads) interstitial fluid is reaching the outer side of the nephrogenic zone, while its inner side is provided via the ends of cortical radiate arteries (big arrow heads). RV: renal vesicle

\section{Formation of Microvessels as Pacesetter for Nephron Induction}

It is not clear, whether delivery is causing in preterm infants alterations of the vascular supply especially on the developing capillaries within the nephrogenic zone. To support provision with nutrition and oxygen in that situation, an actual therapeutic concept is to stimulate formation of capillaries by thymosine $ß-4[11,109]$. The molecule is a peptide consisting of 43 amino acids and is expressed in the developing kidney. Moreover, thymosine $ß-4$ forms a complex with integrin-linked kinase resulting in an activation of survival kinase Akt that finally stimulates production of vascular endothelial growth factor (VEGF). Without interpreting possible side effects, the chances for a therapeutic application are not bad, since synthetic thymosine $\beta-4$ shows a perfect biodistribution [110]. To impede adverse effects on other organs, one possibility is to administer thymosine $\beta-4$ via renal arteries. However, its pharmacological effect on the nephrogenic zone and its ability to stimulate formation of new nephrons was up to date not under solid investigation. One must also consider that a therapeutically increased level of VEGF might be antagonized so that the expression of pigment-epithelium-derived factor (PEDF) in the nephrogenic zone would shift in the focus of actual therapeutic interest [111]. 


\section{In Search of the Main Switch}

VEGF is a special morphogen during kidney development [112]. Beside its impressive angiogenic role, VEGF also stimulates branching of the ureteric bud via VEGF-receptor2 [113]. In culture experiments with rat ureteric buds isolated from organ anlage it was shown that the response is dependent on the dose of administered VEGF. These data place VEGF in an unique position of being principally able to modulate as well vascular development (stroma) as branching of ureteric buds (parenchyma) and in turn induction of nephrons.

However, between organ anlage in animal species and development of fetal parenchyma in a human kidney are differences. For example, switches in the developmental program result in a special pattern of $C D$ ampullae in the nephrogenic zone of a fetal human kidney [20]. Consequently, it is urgent to examine, whether expression of VEGF-receptor2 in the CD ampulla of a late fetal kidney is still the same as it was in the ureteric bud during organ anlage. Moreover, the microenvironment in the nephrogenic zone must be physiological, since hypoxia inhibits nephrogenesis through paracrine VEGF despite its ability to enhance tubulogenesis [114]. Recent data show that VEGF is expressed in the embryonic kidney even in the absence of any external hypoxic stimulus [115].

Moreover, during development of fetal renal parenchyma abundant renin producing cells occur along maturing nephrons and the nephrogenic zone. During proceeding nephrogenesis renin expression shifts in a wavelike pattern from the vessel wall of cortical radiate arteries to terminal portions of developing afferent arterioles on arising glomeruli. Those develop at the lower part of S-shaped bodies [116]. Most interestingly, lack of prorenin receptor (PRR) in the ureteric buds in mice causes reduced branching during development resulting in a decreased nephron endowment [117]. Additionally, intact signaling between renin, prorenin and PRR maintains the nephrogenic stem cell population supporting in turn progression of nephrogenesis [118]. For a human fetal kidney short before birth, it would be therefore important to known, whether renin, prorenin and PRR have a vital influence on stem cell niche activity. In that context one must also analyze, whether renin expression during fetal development of the kidney depends on intact sympathetic innervation and on ß-adrenergic receptor input [119]. Finally, one should bear in mind that in transfilter cultures only embryonic spinal cord and brain were effective, whereas the isolated ureteric bud did not induce tubules in nephrogenic mesenchyme [120].

\section{Drugs for Prolongation of Nephrogenesis}

Before described morphogens belong to highly effective natural molecules that maintain in the nephrogenic zone stemness and promote nephron induction within the niche. However, due to individual biophysical features, uncertain bioavailability and unclear form of administration, it is questionable, whether they can be administered in preterm infants for therapeutic prolongation of nephrogenesis. In this context, also possible side effects of administered morphogens must be investigated. For example, exogenously applied GDNF promotes unwished formation of ectopic ureteric buds [121].
To avoid therapeutic administration of morphogens, a series of other molecules is on the screen, which appears as a viable alternative for prolongation of nephrogenesis. For years lithium $\left(\mathrm{Li}^{+}\right)$is known as a potent mood stabilizer. Actual experiments show in this connection that the ion accumulates in neurogenic brain regions [122]. However, in earlier culture experiments it was demonstrated that $\mathrm{Li}^{+}$ions can also elicit the early stages of epithelial differentiation in isolated nephrogenic mesenchyme [123]. Further $\mathrm{Li}^{+}$ions reduce glycogen synthase kinase-3 activity directly on the molecule and by increasing the inhibitory phosphorylation of the enzyme [124]. In this context, it was shown that not only $\mathrm{Li}^{+}$ions but also 6-bromoindirubin-3'-oxime act as inhibitors on glycogen synthase kinase- 3 stimulating in turn in mesenchyme abundant epithelial differentiation and full segregation of nephrons [125].

Moreover, glycogen synthase kinase-3 inhibition by CHIR99021 promotes self-renewal of embryonic stem cells in mice [126]. Without wanting to interpret ethical issues, for human pluripotent stem cells (PSC) it was shown that incubation with the Wnt pathway activator CHIR99021 in combination either with retinoid AM580 or TTNPB results in renal tubule-like structures [127]. When PSCs were treated by CHIR99021 in combination with fibroblast growth factor 9 and activin, cells could be generated that express markers of intact nephrogenic mesenchyme [128].

Finally, in culture experiments isolated competent nephrogenic mesenchyme can be induced to develop renal tubules. In most of those cases culture medium was used that contained animal serum. Interestingly, application of serum-free respectively chemically defined medium impedes formation of renal tubules. In contrast, supplementation of chemically defined medium (I-MEM) including transferrin leads to effective formation of tubules [129]. Consequently, in the case a too low iron content in the nephrogenic zone of a preterm respectively low birth weight babies is causing impairment of nephrogenesis, administration of transferrin might compensate it [130]. Possibly, a culture model with pieces of isolated but intact nephrogenic zone can help to solve some of the assignments in the initial phase of experimental clearance [131].

\section{Conclusion}

Special microarchitecture of the nephrogenic zone, an incomplete capillary system, aligned stem cell niches including filigree extracellular matrix, a cell to cell communication via tunneling nanotubes and a pleiotropic morphogen action are the challenges, when a therapeutic concept prolonging nephrogenesis in preterm or low birth weight babies is provided. The actual problem is that morphological, physiological, biochemical and pharmacological aspects of the nephrogenic zone in the human fetal kidney were up to date not thoroughly investigated. To obtain the necessary information, basic research by modern ultrastructural, biochemical, physiological and cell biological set ups has to be performed in the next future:

- Unknown is the exact microarchitecture of the nephrogenic zone.

- Not available is an actual analysis of the forming vessel system.

- Unclear is the ultrastructural composition of the renal stem cell niche. 
- Unexplored are physiological parameters of cells in the nephrogenic zone.

- Unidentified is the intrinsic cell communication via tunneling nanotubes.

- Not explored is the transport of morphogens from cell to cell.

- Unacquainted is the therapeutic administration of drugs.

- Unknown are side effects of eligible drugs.

To dampen exaggerated expectations, only a solid base of research built up within the next years will exhibit the path for a secure therapy to prolong nephrogenesis in preterm or low birth weight infants. It is obvious that the pre-clinical environment must closely cooperate with clinicians of neonatal intensive care units, involved pharmacologists and pharmacists. A lot done, a lot more to do - so let us get started!

\section{Acknowledgements}

Project was supported by Emeriti Research Fund (ERF), University of Regensburg, D-93053 Regensburg, Germany.

\section{Conflict of interest}

The author has no conflict of interest.

\section{References}

[1] Reidy KJ, Rosenblum ND. Cell and molecular biology of kidney development. Semin Nephrol. 2009; 29: 321-337

[2] Nagalakshmi VK, Yu J. The ureteric bud epithlium: Morphogenesis and roles in metanephric kidney patterning. Mol Reprod Dev. 2015; 82: 151-166

[3] Faa G, Sanna A, Gerosa C et al. Renal physiological regenerative medicine to prevent chronic renal failure: Should we start at birth? Clin Chim Acta 2015; 444: 156-162

[4] Abitbol CL, De Freitas MJ, Strauss J. Assessment of kidney function in preterm infants: Lifelong implications. Pediatr. Nephrol. 2016; 31: 2213-2222

[5] Luyckx VA, Perico N, Somaschini M et al. A developmental approach to the prevention of hypertension and kidney disease: $A$ report from the low birth weight and nephron number working group. Lancet 2017, pii: S0140-6736(17)30576-7 doi:10.1016/S01406736(17)30576-7

[6] Kandasamy Y, Smith R, Wright IM et al. Extra-uterine growth in preterm infants: Oligonephropathy and prematurity. Pediatr Nephrol 2013; 28: 1791-1796

[7] Stritzke A, Thomas S, Amin $\mathrm{H}$ et al. Renal consequences of preterm birth. Molecular and Cellular Pediatrics 2017; 4: 2

[8] Sanna A, Fanos V, Gerosa C et al. Immunohistochemical markers of stem/progenitor cells in the developing human kidney. Acta Histochem. 2015; 117: 437-443

[9] Noto A, Fanos V, Dessi A. Metabolomics in newborns. Adv Clin Chem 2016; 74: 35-61

[10] Fanni D, Gerosa C, Nemolato S et al. "Physiological" renal regenerating medicine in VLBW preterm infants: Could a dream come true? J Matern Fetal Neonatal Med 2012; 25: 41-48

[11] Fanos V, Castagnola Faa G. Prolonging nephrogenesis in preterm infant: a new approach for prevention of kidney disease in adulthood. Iran J Kidney Dis 2015; 9: 180-185
[12] Saboktakin MR, Tabatabaei RM. Supramolecular hydrogels as drug delivery systems. Int J Biol Macromol. 2015; 75: 426-436

[13] Curtis LM, Chen S, Chen B et al. Contribution of intrarenal cells to cellular repair after acute kidney injury: Subcapsular implantation technique. Am J Physiol Renal Physiol 2008; 295: F310-F314

[14] Rumballe BA, Georgas KM, Combes AN et al. Nephron formation adopts a novel spatial topology at cessation of nephrogenesis. Dev Biol. 2011; 360: 110-122

[15] Fanni D, Sanna A, Gerosa C et al. Each niche has an actor: Multiple stem cell niches in the preterm kidney. Ital J Pediatr 2015; 41: 78

[16] Minuth WW, Denk L. Structural links between the renal stem/ progenitor cell niche and the organ capsule. Histochem Cell Biol 2014; 141: 459-471

[17] Kloth S, Aigner J, Brandt E et al. Histochemical markers reveal an unexpected heterogeneous composition of the renal embryonic collecting duct epithelium. Kidney Int. 1993; 44: 527-536

[18] Park HC, Yasuda K, Kuo MC et al. Renal capsule as a stem cell niche. Am J Physiol Renal Physiol 2010; 298: F1254-F1262

[19] Fanni D, Gerosa C, Vinci L et al. Interstitial stromal progenitors during kidney development: Here, there and everywhere. J Matern Fetal Neonatal Med 2016; 29: 3815-3820

[20] Al-Awqati Q, Goldberg MR. Architectural patterns in branching morphognesis in the kidney. Kidney Int. 1998; 54: 1832-1842

[21] Minuth WW. View onto the nephrogenic zone before stem cell niches come apart: Challenge for smart drug delivery. J Drug Res Dev 2017; 3:http://dx.doi.org/10.16966/2470-1009.127

[22] Combes AN, Davies JA, Little MH. Cell-cell interactions driving kidney morphogenesis. Curr Top Dev Biol. 2015; 112: 467-508

[23] Minuth WW, Denk L, Miess C et al. Peculiarities of the extracellular matrix in the interstitium of the renal stem/progenitor cell niche. Histochem Cell Biol 2011; 136: 321-334

[24] Minuth WW, Denk L. Illustration of extensive extracellular matrix at the epithelial-mesenchymal interface within the renal stem/ progenitor cell niche. BMC Clin Pathol 2012; 12: 16

[25] Schumacher $K$, Strehl R, de Vries U et al. SBA-positive fibers between the CD ampulla, mesenchyme and renal capsule. J Am Soc Nephrol. 2002; 13: 2446-2453

[26] Schumacher K, Strehl R, Minuth WW. Characterization of Micro-fibers at the interface between the renal collecting duct ampulla and the cap condensate. Nephron Exp Nephrol. 2003; 95: e43-e54

[27] Ikeya M, Fukushima K, Kawada M et al. Cv2, functioning as a pro-BMP factor via twisted gastrulation, is required for early development of nephron precursors. Development 2010; 337: 405-414

[28] Minuth WW, Denk L. The interstitial interface within the renal stem/ progenitor cell niche exhibits an unique microheterogenous composition. Int J Mol Sci. 2013; 14: 13657-13569

[29] Qiao J, Cohen D, Herzlinger D. The metanephric blastema differentiates into collecting duct system and nephron epithelia in vitro. Development 1995; 121: 3207-3214

[30] Kanwar YS, Zheng ZL, Kumar A et al. D-glucose-induced dysmorphogenesis of embryonic kidney. J Clin Invest. 1996; 98: 2478-2488

[31] Barasch J, Yang J, Qiao J et al. Tissue inhibitor of metalloproteinase-2 stimulates mesenchymal growth and regulates epithelial branching during morphogenesis of the rat metanephros. J Clin Invest. 1999; 103: $1299-1207$

[32] Strehl R, Trautner $\mathrm{V}$, Kloth $\mathrm{S}$ et al. Existence of a dense reticular meshwork surrounding the nephron inducer in neonatal rabbit kidney. Cell Tissue Res. 1999; 298: 539-548

[33] Strehl R, Minuth WW. Partial identification of the mab (CD)Amp1 antigen at the epithelial-mesenchymal interface in the developing kidney. Histochem Cell Biol 2001; 116: 389-396 
[34] Minuth WW, Denk L. Advanced fixation for transmission electron microscopy unveils special extracellular matrix within the renal stem/ progenitor cell niche. Methods Mol Biol. 2015; 1212: 21-23

[35] Minuth WW, Denk L. Cell projections and extracellular matrix cross the interstitial interface within the renal stem/progenitor cell niche: Accidental, structural or functional cues? Nephron Exp Nephrol 2012; 122: 131-140

[36] Schumacher K, Klar J, Wagner C et al. Temporal-spatial co-localisation of tissue transglutaminase (Tgase2) and matrix metalloproteinase- 9 (MMP-9) with SBA-positive micro-fibers in the embryonic kidney cortex. Cell Tissue Res. 2005; 319: 491-500

[37] Riggins KS, Mernaugh G, Su Y et al. MT1-MMP-mediated basement membrane remodeling modules renal development. Exp Cell Res. 2010; 316: 2993-3005

[38] Zhang Z, Xing J, Gong R et al. Transglutaminase-1 regulates renal epithelial cell proliferation through activation of Stat-3. J Biol Chem. 2009; 284: 3345-3353

[39] Antonyak MA, Li B, Regan AD et al. Tissue transglutaminase is an essential participant in the epidermal growth factor-stimulated signaling pathway leading to cancer cell migration and invasion. J Biol Chem. 2009; 284: 17914-17925

[40] Keillor JW, Apperley KY. Transglutaminase inhibitors: A patent review. Expert Opin Ther Pat 2016; 24: 49-63

[41] Müller $U$, Wang $D$, Denda $S$ et al. Integrin $\alpha 8 \beta 1$ is critically important for epithelial-mesenchymal interactions during kidney morphogenesis. Cell 1997; 88: 603-613

[42] Brandenberger R, Schmidt A, Linton J et al. Identification and characterization of a novel extracellular matrix protein nephronection that is associated with integrin $\alpha 8 \beta 1$ in the embryonic kidney. J Cell Biol. 2001; 154: 447-458

[43] Sato Y, Shimono C, Li S et al. Nephronectin binds to heparan sulfate proteoglycans via MAM domain. Matrix Biol. 2013; 32: 188-195

[44] Uchiyama Y, Sakaguchi M, Terebayashi T et al. Kif26b, a kinesin family gene, regulates adhesion of the embryonic kidney mesenchyme. Proc Natl Acad Sci USA 2010; 107: 9240-9245

[45] Nishinakamura R, Uchiyama Y, Sakaguchi M et al. Nephron progenitors in the metanephric mesenchyme. Pediatr Nephrol. 2011; 26: $1463-1467$

[46] Minuth WW, Denk L. When morphogenetic proteins encounter special extracellular matrix and cell-cell connections at the interface of the renal stem/progenitor cell niche. Anatomy Cell Biology 2015; 48: $1-9$

[47] Domhan S, Ma L, Tai A et al. Intercellular communication by exchange of cytoplasmic material vis tunneling nano-tube like structures in primary human renal epithelial cells. PLoS One 2011; 6: e21283

[48] Krause M, Rak-Raszewska A, Pietilä I et al. Signaling during kidney development. Cells 2015; 4: 112-132

[49] Hilliard SA, Yao X, El-Dahr SS. Mdm2 is required for maintenance of the nephrogenic niche. Dev Biol. 2014; 387: 1-14

[50] Kobayashi A, Valerius MT, Mugford JW et al. Six2 defines and regulates a multipotent self-renewing nephron progenitor population throughout mammalian kidney development. Cell Stem Cell 2008; 3: 169-181

[51] Kopan R, Chen S, Little M. Nephron progenitor cells: Shifting the balance of self-renewal and differentiation. Curr Top Dev Biol. 2014; 107: 293-331

[52] Carroll T], Das A. Defining the signals that constitute the nephron progenitor niche. J Am Soc Nephrol. 2013; 24: 873-876

[53] Michos O, Goncalves A, Lopez-Rios J et al. Reduction of BMP4 activity by gremlin 1 enables ureteric bud outgrowth and GNDF/Wnt11 feedback signaling during kidney branching morphogenesis. Development 2007; 134: 2397-2405
[54] Faa G, Gerosa C, Fanni D et al. Morphogenesis and molecular mechanisms involved in human kidney development. J Cell Physiol. 2012; $227: 1257-1268$

[55] Chai OH, Song CH, Park SK et al. Molecular regulation of kidney development. Anat Cell Biol 2013; 46: 19-31

[56] O'Brien LL, Mc Mahon AP. Induction and patterning of the metanephric nephron. Semin Cell Dev Biol. 2014; 36: 31-38

[57] Oxburgh L, Brown AC, Muthukrishnan SD et al. Bone morphogenetic protein signaling in nephron progenitor cells. Pediatr Nephrol. 2014; 29: 531-536

[58] Brazil DP, Church RH, Surae S et al BMP signalling: Agony and antogony in the family. Trends Cell Biol. 2015; 25: 249-264

[59] Piludu M, Fanos V, Congiu T et al. The pine-cone body: An intermediate structure between the cap mesenchyme and the renal vesicle in the developing nof mouse kidney revealed by an ultrastructural study. J Matern Fetal Neonatal Med 2012; 25: 72-75

[60] Potter SS, Brunskill EW, Patterson LT. defining the genetic blueprint of kidney development. Pediatr Nephrol. 2011; 26: 1469-1478

[61] Kispert A, Vainio S, McMahon AP. Wnt-4 is a mesenchymal signal for epithelial transformation of metanephric mesenchyme in the developing kidney. Development 1998; 125: 4225-4234

[62] Rak-Raszewska A, Hauser PV, Vainio S. Organ in vitro culture: what have we learned about early kidney development. Stem Cells Int 2015; 959807

[63] Lehtonen E. Epithelio-mesenchymal interface during mouse kidney tubule induction in vivo. J Embryol Exp Morph 1975; 34: 695-705

[64] Saxen L, Lehtonen E. Transfilter induction of kidney tubules as a function of the extent and duration of intercellular contacts. J Embryol Exp Morphol 1978; 47: 97-109

[65] Migliorini E, Thakar D, Kühnle J et al. Cytokines and growth factors cross-link heparin sulfate. Open Biol. 2015; 5: pii: 150046

[66] Fairchild CL, Barna M. Specialized filopodia: At the 'tip' of morphogen transport and vertebrate tissue patterning. Curr Opin Genet Dev 2014; 27: 67-73

[67] Lander AD. Morpheus unbound: Reimagining the morphogen gradient. Cell 2007; 128: 245-256

[68] Abuharbeid S, Czubayko F, Aigner A. The fibroblast growth factorbinding protein FGF-BP. Int J Biochem Cell Biol. 2006; 38: 1463-1468

[69] Swencki-Underwood B, Mills JK, Vennarini J et al. Expression and characterization of a human BMP-7 variant with improved biochemical properties. Protein Expr Purif. 2008; 57: 312-319

[70] Pohl TL, Boergemann JH, Schwaerzer GK et al. Surface immobilization of bone morphogenetic protein 2 via self-assembled monolayer formation induces cell differentiation. Acta Biomater. 2012; 8: 772-780

[71] Creanga A, Glenn TD, Mann RK et al. Scube/You activity mediates release of dually lipid-modified Hedgehog signal in soluble form. Genes Dev. 2012; 26: 1312-1325

[72] Gross JC, Boutros M. Secretion and extracellular space travel of Wnt proteins. Curr Opin Genet Dev 2013; 23: 385-390

[73] Bandari S, Exner S, Ortmann C et al. Sweet on Hedgehogs: regulatory roles of heparin sulfate proteoglycans in Hedgehog-dependent cell proliferation and differentiation. Curr Protein Pept Sci. 2015; 16: 66-76

[74] Minuth WW, Denk L. What is the functional background of filigree extracellular matrix and cell-cell connections at the interface of the renal stem/progenitor cell niche? J Pediatric Neonatal Individualized Medicine 2016; 5: e50115

[75] Minuth WW, Denk L. Special morphological features at the interface of the renal stem/progenitor cell niche force to reinvestigate transport of morphogens during nephron induction. BioResearch Open Access 2016; 5: 49-60 
[76] Akiyama T, Gibson MC. Morphogen transport: Theoretical and experimental controversies. Wiley Interdiscip Rev Dev Biol 2015; 4: 99-112

[77] Gheisari Y, Yokko T, Matsumoto K et al. A thermoreversible polymer mediates controlled release of glial cell line-derived neurotrophic factor to enhance kidney regeneration. Artif Organs. 2010; 34: 642-647

[78] Costantini F, Kopan R. Patterning a complex organ: Branching morphogenesis and nephron segmentation in kidney development. Dev Cell. 2010; 18: 698-712

[79] Yan D, Lin X. Shaping morphogen gradients by proteoglycans. Cold Spring Harb Perspect Biol 2009; 1: a002493

[80] Rosines E, Schmidt HJ, Nigam SK. The effect of hyaluronic acid size and concentration on branching morphogenesis and tubule differentiation in developing kidney culture systems: Potential applications to engineering of renal tissues. Biomaterials 2007; 28: 4806-4817

[81] Shah M, Sakurai H, Sweeney DE et al. Hs2st mediated kidney kidney mesenchyme induction regulates early ureteric bud branching. Dev Biol. 2010; 339: 354-365

[82] Shah MM, Sakurai H, Gallegos TF et al. Growth factor-dependent branching of the ureteric bud is modulated by selective $6-0$ sulfation of heparin sulfate. Dev Biol. 2011; 356: 19-27

[83] Nigam SK, Bush KT. Growth factor-heparan sulfate "switches" regulating stages of branching morphogenesis. Pediatr Nephrol. 2014; 29: 727-735

[84] Halt K, Vainio S. Coordination of kidney organogenesis by WNT signaling. Pediatr Nephrol. 2014; 29: 737-744

[85] Luz M, Spanni-Müller S, Özhan G et al. Dynamic association with donor cell filopodia and lipid-modification are essential features of wnt8a, during patterning of the zebrafish. PLoS One 2014; 9: e84922

[86] Stanganello E, Hagemann Al, Mattes B et al. Filopodia-based Wnt transport during vertebrate tissue patterning. Nat Commun 2015; 6: 5846

[87] Hsiung F, Ramirez-Weber FA, Iwaki DD et al. Dependence of Drosophila wing immaginal dis cytonemes on decapentaplegic. Nature 2005; 437: 560-563

[88] Gill PS, Rosenblum ND. Control of murine kidney development by sonic hedgehog and its GLI effectors. Cell Cycle. 2006; 5: 1426-1430

[89] Bischoff M, Gradilla AC, Seijo I et al. Cytonemes are required for the establishment of a normal Hedgehog morphogen gradient in Drosophila epithelia. Nat Cell Biol. 2013; 15: 1269-1281

[90] Sanders TA, Llagostatera E, Barna M. Specialized filopodia direct longrange transport of SSH during vertebrate tissue patterning. Nature 2013; 497: 628-632

[91] Liu Z, Shi H, Szymczak LC et al. Promotion of bone morphogenetic protein signaling by tetraspins and glycosingolipids. PLos Genet. 2015; 11: e1005221

[92] Inaba M, Buszczak M, Yamashita YM. Nanotubes mediate niche-stemcell signaling in the drosophila testis. Nature 2015; 523: 329-332

[93] Gurke S, Barroso JFV, Gerdes HH. The art of cellular communication: tunneling nanotubes bridge the divide. Histochem Cell Biol 2008; 129: $539-550$

[94] Kimura S, Hase K, Ohno $\mathrm{H}$. The molecular basis of induction and formation of tunneling nanotubes. Cell Tissue Res. 2013; 352: 67-76

[95] Gerdes HH, Pepperkok R. Cell-to-cell communication: current views and future perspectives. Cell Tissue Res. 2013; 352: 1-3

[96] Austefjord MW, Gerdes HH, Wang X. Tunneling nanotubes: Diversity in morphology and structure. Comm Integr Biol 2014; 7: e27934

[97] Valente S, Rossi R, Resta L et al. Exploring the human mesenchymal stem cell tubule communication network through electron microscopy. Ultrastruc Pathol 2015; 39: 88-94
[98] Abounit S, Delage E, Zurzolo C. Identification of tunneling nanotubes for intercellular trafficking. Curr Protoc Cell Biol 2015; 67: 12.10.112.10.21

[99] Plotnikov EY, Khryapenkova TG, Galina SI et al. Cytoplasm and organelle transfer between mesenchymal potent stromal cells and renal tubular cells in co-culture. Exp Cell Res. 2010; 316: 2447-2455

[100] Benard M, Schapman D, Lebon A et al. Structural and functional analysis of tunneling nanotubes (TnTs) using gCW STED and gconfocal approaches. Biol Cell. 2015; 107: 419-425

[101] Borges FT, Reis LA, Schor N. Extracellular vesicles: Structure, function, and potential clinical uses in renal diseases. Braz J Med Res 2013; 46: 824-830

[102] Bianchi F, Sala E, Donadei C et al. Potential advantages of acute kidney injury management by mesenchymal stem cells. World J Stem Cells. 2014; 6: 644-650

[103] Camussi G, Deregibus MC, Tetta C. Paracrine/endocrine mechanism of stem cells on kidney repair: Role of microvesicle-mediated transfer of genetic information. Curr Opin Nephrol Hypertens. 2010; 19: 7-12

[104] Aggarwal S, Moggio A, Bussolati B. Concise review: Stem/progenitor cells for renal repair : current knowledge and perspectives. Stem Cells Transl Med 2013; 2: 1011-1019

[105] Holthöfer H. Vascularization of the embryonic kidney. Detection of endothelial cells with Ulex europaeus I lectin. Cell Diff 1987; 20: 27-31

[106] Kloth S, Ebenbeck C, Monzer ] et al. Three-dimensional organization of the developing vasculature of the kidney. Cell Tissue Res. 1997; 287: 193-201

[107] Han KH, Lim JM, Kim MY et al. Expression of endothelial nitric oxide synthase in developing rat kidney. Am J Physiol Renal Physiol 2005; 288: F694-F702

[108] Rymer C, Paredes ], Halt K et al. Renal blood flow and oxygenation drive nephron progenitor differentiation. Am J Physiol Renal Physiol 2014; 307: F337-F345

[109] Nemolato S, Cabras T, Messana I et al. Do $\beta$-thymosins play a role in human nephrogenesis? In: Fa G, Fanos V.eds. Kidney development in renal pathology. Berlin: Springer; 2014: 81-94

[110] Mora CA, Baumann CA, Paino JE et al. Biodistribution of synthetic thymosin beta 4 in the serum, urine, and major organs in mice. Int J Immunopharmacol. 1997; 19: 1-8

[111] Pina AL, Kubitza M, Brawanski A et al. Expression of pigment-epithelium-derived factor during kidney development and aging. Cell Tissue Res. 2007; 329: 329-338

[112] Kitamoto Y, Tokunaga H, Tomita K. Vascular endothelial growth factor is an essential molecule for mouse kidney development: glomerulogenesis and nephrogenesis. J Clin Invest. 1997; 99: 2351-2357

[113] Marlier A, Schmidt-Ott KM, Gallagher AR et al. Vegf as an epithelium morphogen modulates branching morphogenesis of embryonic kidney by direct acting on the ureteric bud. Mech Dev. 2009; 126: 91-98

[114] Schley G, Scholz H, Kraus A et al. Hypoxia inhibits nephrogenesis through paracrine Vegfa despite the ability to enhance tubulogenesis. Kidney Int. 2015; 88: 1283-1292

[115] Halt KJ, Pärssinen HE, Juntilla SM et al. CD146(+) cells are essential for kidney vasculare development. Kidney Int. 2016; 90: 311-324

[116] Machura K, Steppan D, Neubauer B et al. Developmental renin expression in mice with defective renin-angiotensin system. Am J Physiol Renal Physiol 2009; 297: F1371-F1380

[117] Yosypiv IV. Renin-angiotensin system in the ureteric bud branching morphogenesis: Implications for kidney disease. Pediatr Nephrol. 2014; 29: 609-620 
[118] Song R, Preston G, Kidd L et al. Prorenin receptor is critical for nephron progenitors. Dev Biol. 2016; 409: 382-391

[119] Neubauer B, Machura K, Schnermann J et al. Renin expression in large renal vessels during fetal development depends on functional beta $1 /$ beta2-adrenergic receptors. Am J Physiol Renal Physiol 2011; 301: F71-F77

[120] Sariola S, Ekblom P, Henke-Fahle S. Embryonic neurons as in vitro inducers of differentiation of nephrogenic mesenchyme. Dev Biol. 1989; 132: 271-281

[121] Ola R, Jakobson M, Kvist J et al. The GDNF target Vsnl1 marks the ureteric tip. J Am Soc Nephrol. 2011; 22: 274-284

[122] Zanni G, Michno W, Di Martino E et al. Lithium accumulates in neurogenic brain regions as revealed by high resolution ion imaging. Sci Rep 2017; 7: 40726

[123] Davies JA, Garrod DR. Induction of early stages of kidney tubule differentiation by lithium ions. Dev Biol. 1995; 167: 50-60

[124] Jope RS. Lithium and GSK-3: one inhibitor, two inhibitory actions, multiple outcomes. Trends Pharmacol Sci. 2003; 24: 441-443

[125] Kuure S, Popsueva A, Jakobson M et al. Glycogen synthase kinase-3 inactivation and stabilization of beta-catenin induce nephron differentiation in isolated mouse and rat kidney mesenchymes. J Am Soc Nephrol. 2007; 18: 1130-1139
[126] Ye S, Tan L, Yang R et al. Pleiotropy of glycogen synthase kinase-3 inhibition by CHIR99021 promotes self-renewal of embryonic stem cells from refractory mouse strains. PLoS One 2012; 7: e35892

[127] Araoka T, Mae S, Kurose $Y$ et al. Efficient and rapid induction of human $\mathrm{iPSCs/ESCs}$ into nephrogenic intermediate mesoderm using small molecule based differentiation methods. PLoS One 2014; 9 : e84881

[128] Lam AQ, Freedman BS, Morizane R et al. Rapid and efficient differentiation of human pluripotent stem cells into intermediate mesoderm that forms tubules expressing kidney proximal tubular markers. J Am Soc Nephrol. 2014; 25: 1211-1225

[129] Thesleff I, Ekblom P. Role of transferrin in branching morphogenesis, growth and differentiation of the embryonic kidney. J Embryol Exp Morphol 1984; 82: 147-161

[130] Li JY, Paragas N, Ned RM et al. Scara5 is a ferritin receptor mediating non-transferrin iron delivery. Dev Cell. 2009; 16: 35-46

[131] Minuth WW. The rabbit nephrogenic zone in culture: past, present and future as a model to investigate causes of impaired nephrogenesis. J Pediatric Neonatal Individualized Medicine 2017; 6: e060111 